\title{
Decision Support System for Strengthening Medium and Short Span Concrete Bridges
}

\author{
Yiqiang Xiang ${ }^{1,2, a}$, Chaochao He ${ }^{1, b}$ Cheng Xing ${ }^{1, c}$, Yuan Xing ${ }^{3, d}$ \\ ${ }^{1}$ Department of Civil Engineering, Zhejiang University, Hangzhou, 310058, China ,
}

2 Cyrus Tang Center for Sensor Materials and Applications, Zhejiang University, Hangzhou 310058, China,

${ }^{3}$ Huahui Engineering Design Group Ltd. Co. Shaoxing, 312000 , China

axiangyiq@zju.edu.cn, ${ }^{b} 21312073 @ z j u . e d u . c n,{ }^{C}$ zhangcheng1006@163.com,

dxingyuan1393@163.com

Keywords: Medium and short span, Bridge, Reinforcing, Decision, Software Development

Abstract. According to the requirement analysis and current main diseases of medium and short span bridges, the decision-making system from preliminary design of reinforcement scheme, hierarchy analysis to comprehensive evaluation and optimization decision models for strengthening medium and short span bridges are studied and developed on the platform of Windows using object-oriented programming method. It has a good man-machine dialogue interface. It can conveniently and objectively assess the project alternatives of strengthening and reconstructing medium-short span reinforced concrete bridges. It has a significant meaning in reality.

\section{Introduction}

In the practical bridge reinforcement project design, the comparison and selection of strengthening schemes is the key to ensure the quality and economy of strengthening work. In fact, this is a process of decision optimization. The its purpose is to choose the better scheme from a number of possible reinforcement schemes so as to achieve the expected goals by the feasible technique, reliable effect, reasonable economy, convenient construction and guaranteed-quality. At present, most of decision making on bridge strengthening only focus on a special goal (e.g. the reinforcement effect, cost, or construction period etc.) to evaluate and guide the engineering practice. However, the goodness and poorness of reinforcement schemes is usually influenced by the multi-factors. Therefore, a research and development of a strengthening strategy support system with a good human-computer dialogue, comprehensive evaluation, designed for medium and short span concrete girder bridges has significant meaning for both theory and engineering. Based on the diseases and cause analysis of the China's superstructure, substructure in medium and short span concrete bridges[1-3], this paper further propose an index evaluation system and reinforcement decision methods referring to the general reinforcement strategies, successful experience, the specific situation of different bridges and relevant codes of bridges. The decision making system for medium and short span concrete bridges reinforcement is also initially researched and developed on the Windows platform.

\section{System Overview}

The system is designed from the user's point of view. Through the summary of bridge diseases, 
strengthening methods and the data entered by users, the decision support system will provide the most convenient and flexible options. There are two specific functions which will be provided in the system. The first one is to set up bridge diseases and strengthening schemes database and data management system while another is to establish the decision-making system for strengthening schemes. Through this work, intelligent and scientific selections of bridge reinforcement scheme problems can be figured out.

This software is designed as stand-alone software under the Windows operating system by using Visual Basic 6.0 as development software and Access as backend database. There are no special requirements on the hardware environment, desktop or laptop computer will all work. However, an administrator with basic knowledge of computer basics and bridge management is required.

\section{Design of System Development}

Database Design. Optimization and decision making system for reinforcing concrete bridges is a comprehensive problem involving bridge inspection, disease assessment, design of reinforcement methods and economics. Firstly, the position and characteristics of diseases should be defined in advance based on the specific detection information on the bridge followed by a summary into disease databases, and its impact on the bridges. Secondly, according to different diseases types and descriptions, the corresponding reinforcement countermeasure will be set up. Certain logic exists between the disease database and the reinforcement countermeasures, which can be displayed using the form of a reinforcing countermeasures tree. The administrator can sort out appropriate solutions to a disease by inputting the position of the bridge disease and a simple description.

During the process of the system database design, logical connections between the disease database library and strengthening measures will be made using the establishment of disease number field, which makes it more convenient to inquire disease and transfer data between the reinforcement scheme tables accordingly.

Interface Design. Visual Basic ${ }^{[2]}$, assisting with environment development, is an event-driven programming language developed by Microsoft. It is one of the major application development environments for Windows platforms. It provides fast implements and a good graphical user interface. By using DAO, RDO, ADO or creating ActiveX controls, it can easily connect to the database, which is the reason it is applied in this system development. Among them, the key points of the development is to apply the interface switch (Tab controls) applications throughout the process of decision-making modules, so that all calculated variables will not be released in the decision-making module process and achieve a real-time call for data.

Decision Making Algorithm. By comparing the advantages and disadvantages of various assessment methods, evaluation methods are determined to be Fuzzy Analytical Hierarchy Process(FAHP) in the decision assessment model. Specific steps are as follows:

Due to the complexity of different issues, each factor that may influence the reinforcing effects is divided into several levels according to the extent of the property. In the qualitative objective universe $[0,1]$, assessed values can be given. Each index in Evaluation system plays a different role in scheme optimization. The importance of each factor is not the same. According to AHP, multiple comparisons between each index on one level can determine its importance. Score the results of the comparison , judgment matrix $\mathrm{p}$ will be obtained with $\mathrm{n}$ rows, $\mathrm{n}$ columns and $u_{n n}$ as elements.

After obtaining the judgment matrix, orders of judgment matrix weights need to be calculated. 
Figuring out the eigenvector corresponding to the maximum characteristic root, which is the order of importance of each evaluation factor, that is, weighted apportionment, constitute the weight set. Weights can be calculated by referring to $\mathrm{Gao}^{[4]}$ and Wang ${ }^{[5]}$.

The fuzzy comprehensive evaluation, calculation and analysis work can be realized and accomplished in Visual Basic and Matlab environment [6-7].

\section{Design of System Function}

The functions of the software can be divided into internal function and external function. The external function refers to an interactive environment which reveals both the input and output of the data. The internal function is the process of data in the software including the storage of information in the database and the analysis of data operation.

The design consists of 8 function modules: the input of disease information, reinforcement scheme, disease analysis, decision support, inquiry, data printing and instructions. All the modules are switched by the interface and the interaction is based on the shared project database. (It uses the database engine through the database access interface method defined by the operating system.) And the design of the system functions is carried out bounded by 8 modules presented above, and in the practical procedure it will be refined so that the ultimate function can be realized in the development tool. At the same time, an operable interface platform will be built for the clients. The followings are the instructions of the function of each module.

Information input. It is required to import the "bridge name", "bridge category" and "span" information in the message box. And the information imported into the "span" should be in the form of a number, otherwise a type error will appear. To achieve the correspondence with the information in database, disease position and type of disease should only be chosen from the drop-down list. When you choose one object in the list, the computer will match it with the field in the database automatically. And the disease number will be presented in the "disease number" message box (Import of information is not allowed in this message box, it can only be ensured by the searching result of the disease position and type of disease). Once the disease number is clear, the alternative schemes can be searched in the database by a click of "Searching for reinforcement scheme" and the result will be saved in the database. If click on the button of "Return to the primary menu", it will exit the decision support module and return to the primary menu.

Primary selection of alternative schemes. Based on the given information, the software will search for the alternative schemes. And the results will be presented in the check box; where the decision maker can choose one. Here we will illustrate it with the example of coping crack. Five alternative schemes can be gotten and we select first four schemes. Click on the "Next", the system will save the schemes chosen in the database for further use. If you click the button "Back", you will return to the "Information input" to revise the bridge disease information. Then, if you choose the button of "Return to the primary menu", you will get out of the decision support module and return to the primary menu.

Evaluation index, evaluation of index membership degree, index membership degree matrix. These three steps are key steps for creating the matrix of index membership. Firstly, the index evaluation module shows evaluation index system defaulted. According to the evaluation index system, index membership degree of each reinforcement scheme is under evaluation. Then, in the "membership degree evaluation", policymakers need to score each index by seven factors according to dropdown box. During the running time of "index membership matrix" module, the system will transform scores into a variable matrix that can be identified and operated by Matrix VB module. Deposited in variable matrixes $R_{1}-R_{4}$, it can be used for sorting calculation of each reinforcement 
scheme.

Weight Calculation. This module aims at confirming the weight of the first the second level index to form the weight vector $A_{1}-A_{4}$ and $A$. Firstly, the system will grade (1-10) the importance level among the index $u_{11}, u_{12}, u_{13}, u_{14}$ in the first level index $U_{1}$ and then it will form a related matrix. Click the "consistency check", the consistency of the membership degree of $p_{1}$ will be checked. Then the project will calculate the weight vector $A_{1}$ or you have to revise $p_{1}$ until it fits with the consistency check. Similarly, the system will work out $A_{2}-A_{4}, A$, and values will be given to the variable $A_{1}-A_{4}, A$ respectively.

Display of the best scheme. According to the maximum membership degree principle, the scheme corresponding to the max value among B1、B2、B3 and B4 will be the best comprehensive scheme. While the scheme corresponding to the max value among $b 1, b 2 、 b 3$ and $b 4$ will be the best scheme under a particular index.

\section{Conclusions}

This article determined the index system and weight of each index for reinforcing medium-short span concrete bridges by FAHP and proposed the multi-level, multi-objective fuzzy comprehensive evaluation model. It made the selection of medium-short span reinforced concrete bridges reconstruction project alternatives be more comprehensive and objective. Meanwhile, it also provided the reinforcement and decision making for bridges with a scientific basis.

With a combination of main diseases of medium and short span bridges, reinforcement schemes, hierarchy analysis, fuzzy comprehensive evaluation and optimization decision model, Decision Support System for Reinforcing Medium and Short Span Concrete Bridges was researched and developed on the platform of Windows by using object-oriented programming method. This system has a good interactive interface. Considering a variety of evaluation indexes, it has certainly theoretical significance and practical values for the scheme selection of reinforcing bridges.

\section{Acknowledgements}

This work was financially supported by cooperation foundation of Zhejiang University-Huahui Eng. Design Group Ltd. Co. and Cyrus Tang Foundation.

\section{Reference}

[1] Y.Q. Xiang, X. Zhang, X. W. Ye. Diseases Analysis and Strengthening of China's Concrete Bridge Superstructure, The 7th International Conference on Bridge Maintenance, Safety and Management, Shanghai, China, July 7-11, 2014

[2] X.Y. He, Y.Q. Xiang, C. Xing. Diseases Analysis and Strengthening of China's Concrete Bridge Substructure, The 7th International Conference on Bridge Maintenance, Safety and Management, Shanghai, China, July 7-11, 2014

[3] C. Xing: Theoretical Study of the Strengthening Methods and Developing of Decision Support System of the Medium/short Span Concrete Beam Bridge. Zhejiang University, 2013.6. (in Chinese) 
[4] H. Z. Gao: Fuzzy Evaluation Method in Municipal Bridge Strengthening Program Optimization. Shanghai University, 2007 (in Chinese)

[5] Z. J. Wang: Optimization of Reliability Assessment and Strengthening Scheme for Bridge in Service. Changan University, 2004 (in Chinese)

[6] X. Z. Dang: Decision of Rehabilitation Programs to Strengthen the Bridge Based on Fuzzy Theory. Zhengzhou University, 2009 (in Chinese)

[7] S. L. Wang, J. Gao \& X. D. Sun: MATLAB Mixed Programming and Engineering Applications. Tsinghua University Press, Beijing, 2008 (in Chinese) 